\title{
Latency Reduction by Dynamic Channel Estimator Selection in C-RAN Networks Using Fuzzy Logic
}

\author{
Ali M. Mahmood ${ }^{(1),(2)}$, Adil Al-Yasiri ${ }^{(1)}$, Omar Y. Alani ${ }^{(1)}$ \\ ${ }^{(1)}$ University of Salford, Manchester, United Kingdom, ${ }^{(2)}$ University of Technology, Iraq \\ a.mahmood7@edu.salford.ac.uk, a.al-yasiri@salford.ac.uk, o.y.k.alani@salford.ac.uk
}

\begin{abstract}
Due to a dramatic increase in the number of mobile users, operators are forced to expand their networks accordingly. Cloud Radio Access Network (C-RAN) was introduced to tackle the problems of the current generation of mobile networks and to support future 5G networks. However, many challenges have arisen through the centralised structure of C-RAN. The accuracy of the channel state information acquisition in the C-RAN for large numbers of remote radio heads and user equipment is one of the main challenges in this architecture. In order to minimize the time required to acquire the channel information in C-RAN and to reduce the end-to-end latency, in this paper a dynamic channel estimator selection algorithm is proposed. The idea is to assign different channel estimation algorithms to the users of mobile networks based on their link status (particularly the SNR threshold). For the purpose of automatic and adaptive selection to channel estimators, a fuzzy logic algorithm is employed as a decision maker to select the best SNR threshold by utilising the bit error rate measurements. The results demonstrate a reduction in the estimation time with low loss in data throughput. It is also observed that the outcome of the proposed algorithm increases at high SNR values.
\end{abstract}

Keywords-C-RAN; Virtual Base Station; Channel Estimation Algorithms; Channel Information

\section{INTRODUCTION}

Recently some research studies have shown that a 5G network is supposed to support 1 million User Equipment (UE) per square kilometre with $1 \mathrm{~ms}$ end-to-end latency [1]. These crucial requirements necessitate the need to develop new technologies to satisfy the intended services demand for a large number of subscribers. To maintain network scalability, cost reduction and higher spectrum efficiency, Cloud Radio Access Network (C-RAN) is one possible, and promising, architecture for mobile networks of the $5 \mathrm{G}$ future [2]. The innovative idea in this architecture is to aggregate the baseband units from multiple base stations into the cloud to gain the advantages of cloud computing features in cellular networks [3]. Whilst using CRAN in mobile networks brings many benefits, as clarified in [4], there are several technical problems associated with it. The overhead of acquiring the Channel State Information (CSI) is one of these problems [5] [6]. This is used to convey the communication links state between the UE and the Remote Radio Heads (RRHs). The challenge with CSI is that it changes in a fast and dynamic manner with the change in the communication channel conditions and the mobility of the UE as well. In dense networks, like the fully centralised C-RAN, interchange of the CSI to adapt the communication channel is accompanied with a rise in the burden of high computational complexity [7]. However, inaccuracy in the acquired CSI affects the whole performance of the cellular network and the data throughput is reduced. In fact, in C-RAN, the sources of the imperfection in the CSI can be classified into three main sources. First, from the computational complexity of processing extremely large channel matrices for all the connected RRHs with their UEs. The second reason for inaccuracy of the CSI may originate from a communication delay. And finally the high overhead of the channel estimation algorithm itself can be considered as one of the sources of imperfection of this information.

The problem of CSI acquisition in C-RAN has been studied extensively, with the literature identifying different solutions to address the challenge of inaccuracy of the channel information in C-RAN architecture. The proposed approaches were: sparsification techniques, the methods of antenna selection, the algorithms of clustering, the approaches of overhead reduction in the fronthaul, and the complexity reduction techniques for the channel estimation algorithms. The following paragraph summarises these briefly.

Sparsification is a technique followed by the studies in [8] [6] [9] for the purpose of making the channel matrix $(\mathrm{H})$ sparser, by excluding a group of the entries from the $\mathrm{H}$ to reduce the computation overhead of acquiring CSI. While the antenna selection techniques are used to select a set of antennas to minimise the acquisition overhead of the CSI as shown in [10] [11] [12]. The methods in [13] [14] [15] [16] have developed to implement clustering techniques in dense networks to reduce the CSI estimation overhead. Hence, in the large network, CSI acquisition is managed by the dimension of cluster, instead of the entire network.

Regarding the approaches of the fronthaul overhead reduction, the overhead that is aggregated at the centralised pool of the Virtual Base Stations (VBSs) comes from a large number of RRHs which are linked to one centralised site. A number of research studies have been conducted to reduce the overhead of the data on the fronthaul link, which are mainly focused on the data compression methods [17] [18] [19]. Another research direction is that there are large numbers of researchers that have tried to minimise the complexity of the channel estimation algorithm itself. This involves either suggesting new estimators or modifying the current estimators as demonstrated in [20] [21]. 
Finally, some methods have been used to reduce both the CSI latency and interference simultaneously, for instance, the authors in [22] introduced Coordinated Fractional Frequency Reuse (Cloud-CFFR) within the context of C-RAN by applying the Coordinated Multi-Point (CoMP) technology only on the UEs at the cell-edge region, to minimise the overall complexity and latency, while minimising the interference at the same time. In addition, the technique of Dynamic Joint Processing (DJP) can also be used in C-RAN to reduce both the interference and latency, as shown in [23], where, both the intra- and inter-cluster interference can be minimised without increasing the size of clusters.

This paper proposes a hybrid channel estimation algorithm in C-RAN. The selection criteria depends on the link condition of the UE. This can be achieved by allocating the users that have high Signal-to-Noise Ratio (SNR) to one estimator, while the low SNR UEs are assigned to another estimation algorithm. The benefit is to reduce the Estimation Time (ET) of the CSI in CRAN. This is due to the fact that some estimators, such as the Minimum Mean Square Estimator (MMSE), has a noticeable ET overhead compared to other estimation algorithms in acquiring CSI. Throughout this study, the most common estimation algorithms, MMSE and the Least Square (LS) estimators, are used as case study scenarios.

The motivation and the contribution of this paper can be summarised as follows. In C-RAN architecture, the high computation overhead of the channel estimator can be considered one of the sources of imperfection in CSI acquisition. Inaccuracy in the CSI can lead to inaccurate decision-making at the VBSs and the network throughput is consequently reduced. This necessitates the need to find new techniques to improve the accuracy of the CSI acquisition. Furthermore, some channel estimators have low computational complexity, such as the LS estimator, but can provide good performance at high SNR [24].

The contribution of this study is represented by developing an algorithm for hybrid channel estimation which can utilise the merits of the two most common estimators (MMSE and LS) to increase the performance of the C-RAN architecture by reducing the time of CSI acquisition. This technique could be a promising approach, especially in future $5 \mathrm{G}$ networks. The UE will have higher SNR due to the structure of 5G, which will deploy large numbers of RRHs and small cells to be closer to the UEs for the purpose of reducing the latency and improving the capacity. Fuzzy logic is employed for the purpose of automatic selection of the SNR threshold to attain accurate results, in balance between the estimation time gain and the throughput loss.

The remaining sections of this research are structured as follows: section II discusses the background to the components of this work. Section III gives a brief description of the research problem. Section IV introduces the algorithm of hybrid channel estimators. Section V illustrates the results of the system with the proposed algorithm along with the discussion. Lastly, section VI demonstrates the conclusions and recommendations for future research.

\section{RESEARCH BACKGROUND}

The key components of this work are discussed in this section, including the architecture of C-RAN, CSI in mobile networks, channel estimation algorithms and the process of link adaption.

\section{A. C-RAN Architecture}

The integration of cloud computing into the mobile environment leads to a novel concept of C-RAN [25]. C-RAN is a promising architecture to achieve an unprecedented system capacity and it has been proposed as one of the potential architectures for 5G networks [26-28]. Unlike the current networks, which have the baseband unit co-located within the cell site, baseband processing in C-RAN is moved to cloud computing for central processing and management.

In comparison with the Distributed RAN (D-RAN) LTE/LTE-A architecture, C-RAN has a number of features that could support the current and the future wireless cellular networks, such as lower costs and energy consumption [4]. CRAN can efficiently address the so called "tidal effect", which is generated in specific crowded areas throughout particular periods. In addition, it provides fast system roll-out via deploying light-weight RRHs as small cells. Unlike the traditional structure of a base station, which requires a mast and housing for the BBU, the RRHs need only the space for the antenna and access to the fronthaul communication link.

In the related literature, $[4,29,30]$, the letter ' $C$ ' of this architecture is defined as meaning Cloud for real time operation, Centralised processing and management, Collaborative radio, or Clean technology, all depicting positive attributes. The concept of this architecture was first proposed in [31] and thoroughly demonstrated in [29]. It consists of three main components, as illustrated in Fig.1 [13], including: i) the RRH which is deployed as the access point to connect wireless UEs just like the traditional networks; ii) the fronthaul optical connection layer between the VBS and RRH, which provides high bandwidth capacity and low latency; iii) the VBS or the Base Band Unit (BBU) pool which is sited in the cloud for the purpose of dynamic processing and allocating resources based on network demand [13].

\section{B. Channel Information in Mobile Networks}

In the present LTE systems, the CSI contains three types of channel information which convey the status of the communication channel between the UE and the RRH. In CRAN, the CSI is calculated by the UEs and transmitted to VBSs to perform the link adaptation in the cloud. The scheduler of each VBS can then decide to adapt the link condition for different UEs or not. These three reports involve the Channel Quality Indicator (CQI), the Rank Indication (RI) and the Precoding Matrix Indicator (PMI) which are together known as CSI [32]. The accuracy of the CSI has a crucial effect on the performance of the entire mobile network, particularly the data throughput (TP) as shown in Fig.2. 


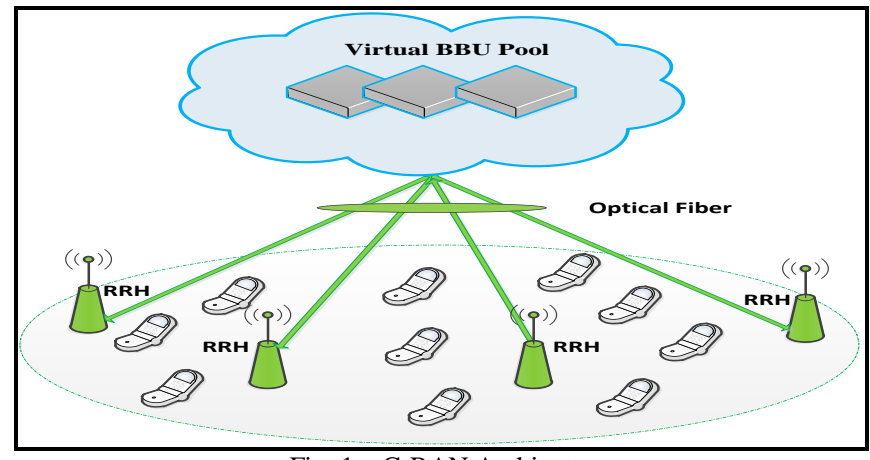

Fig. 1. C-RAN Architecture

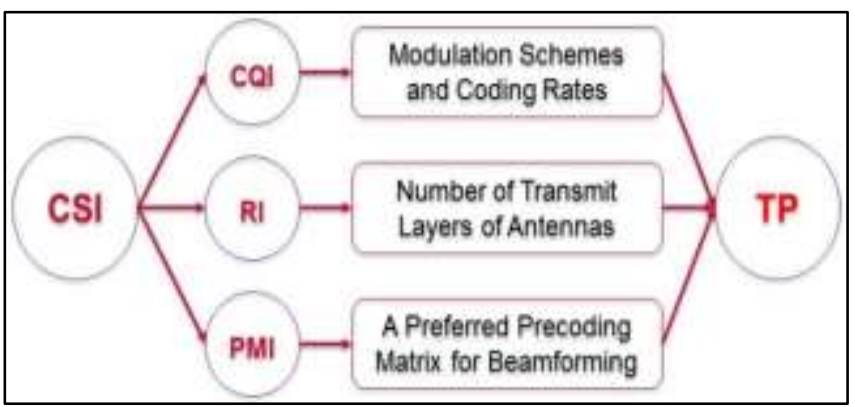

Fig. 2. Relation between the CSI and the data throughput

\section{Channel Estimation Algorithms}

The channel model of the received signal in C-RAN architecture for the uplink transmission is presented in equation (1) for N single antenna RRHs and K single antenna UEs [14], [33], [34].

$$
\boldsymbol{Y}=\boldsymbol{H} \boldsymbol{P}^{\frac{1}{2}} \boldsymbol{X}+\boldsymbol{Z}
$$

where, $\boldsymbol{Y} \in \mathbb{C}^{N x 1}$ is vector of the received signal at the RRHs. $\boldsymbol{H} \in \mathbb{C}^{N x \mathrm{~K}}$ denotes the matrix of channel coefficients between the kth UE and the nth RRH. $\boldsymbol{P} \in \mathbb{R}^{K x \mathrm{~K}}$ is the matrix of transmitting power for each UE. $\boldsymbol{X} \in \mathbb{C}^{K x 1}$ represents the vector of transmitted signal. $\boldsymbol{Z}$ is $(\mathrm{N} \times 1)$ vector of additive Gaussian noise received by the RRHs, $\boldsymbol{Z} \sim \mathcal{C} \mathcal{N}\left(0, N_{0}\right)$. The MMSE and the LS estimator, as the two most commonly used, have the function of estimating channel information (Matrix $\mathrm{H}$ ), which includes the CSI. The following two sub-sections present a brief description for both estimators.

\section{i) Least Square Estimator}

The objective of the channel LS estimator is to minimise the square value between the received signal $\mathrm{Y}$ and the pilot signal $X$. The least square estimate of the channel can be obtained by dividing the received signal by its expected value, as shown in equation (2) $[24,35,36]$. The LS estimator has low computational complexity, since it is designed to work without any knowledge of the statistics of the channels. However, this estimator suffers from performance degradation due to the high mean square error (MSE) [24, 37] in comparison with the MMSE, as shown in Fig.5.

$\widehat{H}_{L S}=\left[\frac{Y}{X}\right]^{T}=X^{-1} Y$

\section{ii) MMSE Estimator}

The MMSE estimator performs second-order statistics to minimise the MSE. The MMSE estimate of the channel responses, as given in equation (3) can be obtained as follows $[24,35,36]$.

Then, the estimation of the channel matrix in the MMSE estimator can be determined as follows:

$\widehat{H}_{m m s e}=R_{H H}\left[R_{H H}+\delta_{n}^{2}\left(X X^{H}\right)^{-1}\right]^{-1} X^{-1} Y$

where, $R_{H H}$ : is the auto-covariance matrix of $\mathrm{H}$.

\section{D) The Process of Link Adaptation}

Link adaptation simply represents the processes of adapting the transmission parameters to reflect instantaneous channel conditions that are currently measured by the system. The precise purpose is to achieve the desired spectral efficiency in the mobile network continuously [7]. The network parameters that are supposed to change dynamically involve: Modulation and Coding Schemes (MCSs), transmission power, the system bandwidth, MIMO transmission modes, the number of transmission layers and the precoding matrix.

In order to support dynamic adaptation to these parameters, the three reports (CQI, PMI and RI) should be generated by the mobile terminal UE and then after receiving this information by the RRHs, it is transmitted to the base stations to aid with scheduling and link adaptation. It is worth mentioning that these reports are transferred between the UE and the RRH by the Physical Uplink Control Channel (PUCCH) in uplink operations and using the Physical Downlink Control Channel (PDCCH) in downlink operations. There are five steps that should be performed to achieve the process of link adaptation in mobile networks, as shown below [7] and in Fig. 3 in detail.

Step 1: Initially at the base station, the downlink transmitter formulates a resource grid of user data from Physical Downlink Shared Channel (PDSCH) and the Downlink Control Information $(\mathrm{PDCCH})$ to send them to the UE.

Step 2: At the mobile terminal, after receiving the resource grid, the UE implements a number of channel quality measurements. The measurements are made up of three reports: CQI, PMI and RI.

Step 3: The UE then inserts the current channel measurements inside the PUCCH to send them back to the base station as a close loop feedback.

Step 4: The base station receives the PUCCH information by the RRHs, then, based on this information, the scheduler decides if the parameters of the next frame of downlink transmission should be updated, or not.

Step 5: The base station then starts to repeat the process for the next frame $(n+1)$, but now embeds the PDCCH with the new scheduling information, which was adapted based on the last frame (n).

Where, $\widehat{H}_{L S}$ denotes the estimate of channel $\mathrm{H}$ 


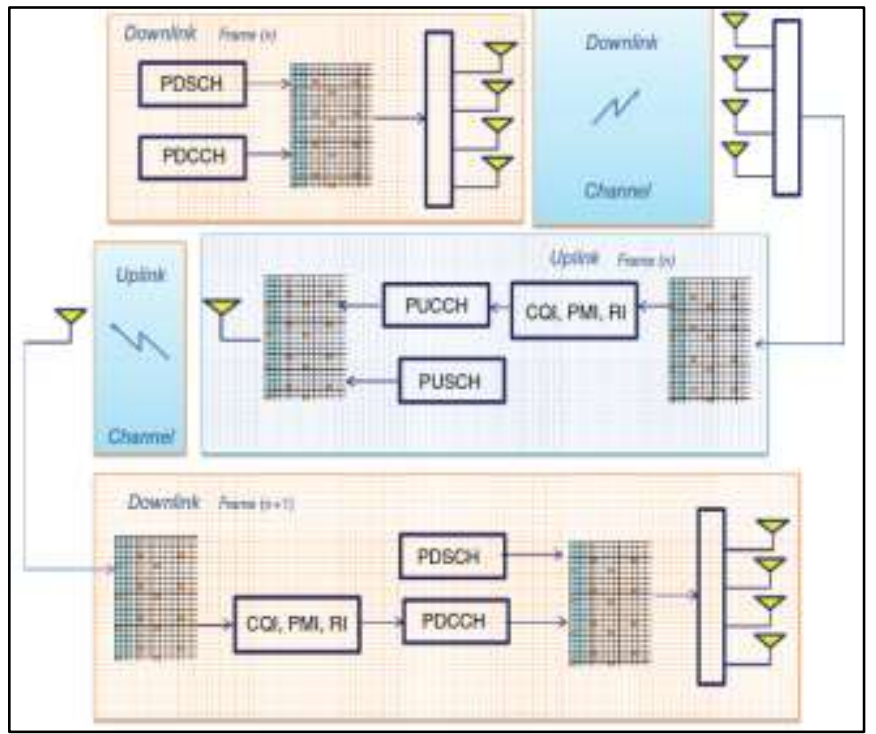

Fig.3. Process of link adaptation [7]

\section{PROBLEM DEFINITION}

This section discusses the main issues which drive the idea of using the mixed estimation algorithm. Firstly, the computation overhead and complexity of channel estimators is illustrated, and secondly, the potentially high SNR values in the next generation networks.

\section{A. The performance of the channel estimators:} estimation time and system data throughput

The main function of the channel estimation algorithm is to acquire or estimate the CSI from the received signal to use this information afterwards, such as in scheduling and link adaptation processes. The challenge is that the process of estimation and link adaptation must be within a timescale called the Coherence Time (CT). If the time of acquiring CSI exceeds $\mathrm{CT}$, then the outdated CSI results in inadequate decision-making at the VBS as the recently received CSI is already delayed and it may not match the present status of the UE.

There are several channel estimation algorithms which have been used in wireless communication systems. After reviewing these algorithms, it can be seen that both the LS and MMSE channel estimation algorithms are of particular interest, due to the fact that they are used in a large number of studies either independently or in combination with other techniques. It has also been observed that there is a trade-off between the performance, in terms of accuracy, and the computation complexity of the estimator. The MMSE estimator has a higher performance than the LS in terms of data throughput and the MSE but it has more computational capacity than LS due to the complexity of the channel covariance matrix inversion [24]. We have conducted a simulation test to verify the performance of both estimators for $1 \times 1$ antenna system. Figures 4,5 and 6 demonstrate clearly the trade-off between the performance and the complexity of both MMSE and LS estimators.

The system with MMSE has superior data throughput response but at the cost of higher computation complexity, as shown in Fig 5. It is worth stating that the overhead of estimation time of the MMSE estimator, as shown in Fig. 5, is relatively modest for $1 \mathrm{x} 1$ antennas system compared to the large number of antennas, as shown in Figures 7 and 8. The result in Fig. 7 shows the growth of estimation time overhead when increasing the number of RRHs in an exponential manner. To quantify the percentage of change (the amount of increase in the estimation time), the result in Fig. 8 illustrates that there is a significant increase in the percentage of latency with the rise in the number of antennas/RRHs at the VBS. For instance, with $128 \times 4$ antennas system, the latency is increased almost 700 times compared to the latency of $1 \mathrm{x} 1$ antenna system.

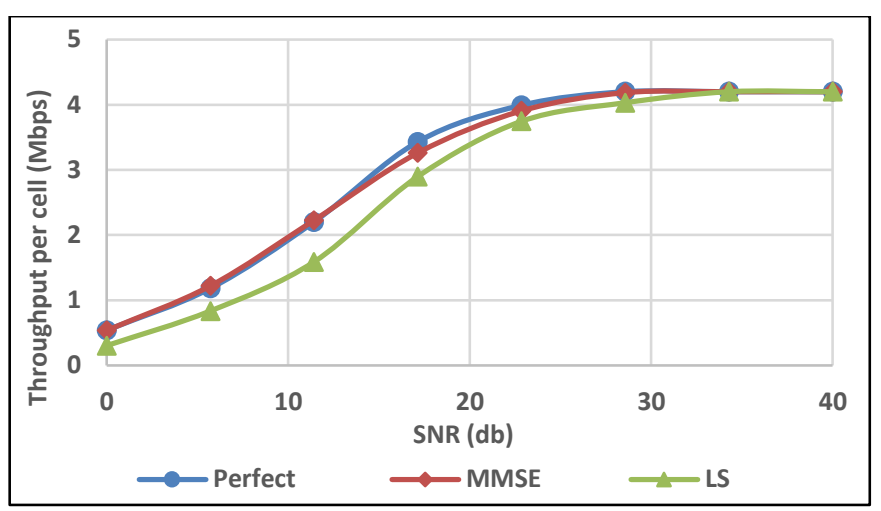

Fig. 4. Comparison between the performances of estimation algorithms for 1 Antenna UE x 1 Antenna VBS

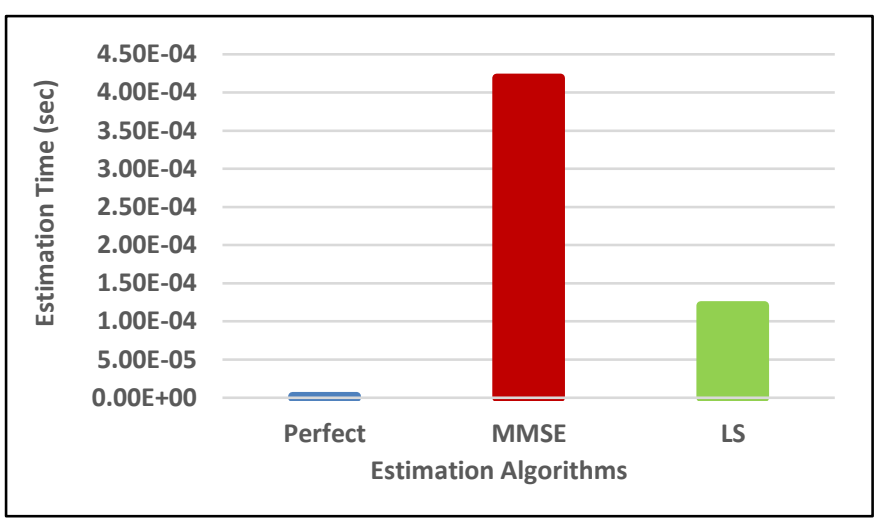

Fig. 5. Comparison between MMSE and LS in terms of estimation time for 1 Antenna UE x 1 Antenna VBS

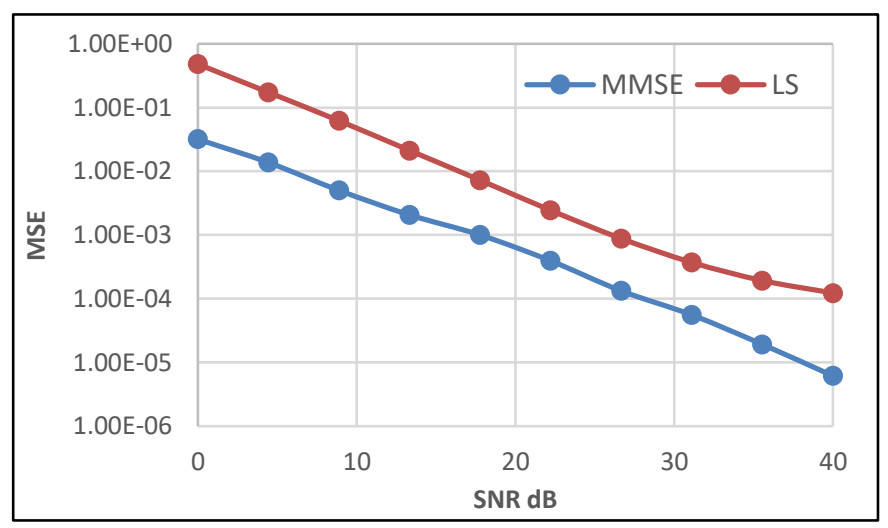

Fig.6. Comparison between MMSE and LS estimators in terms of MSE 


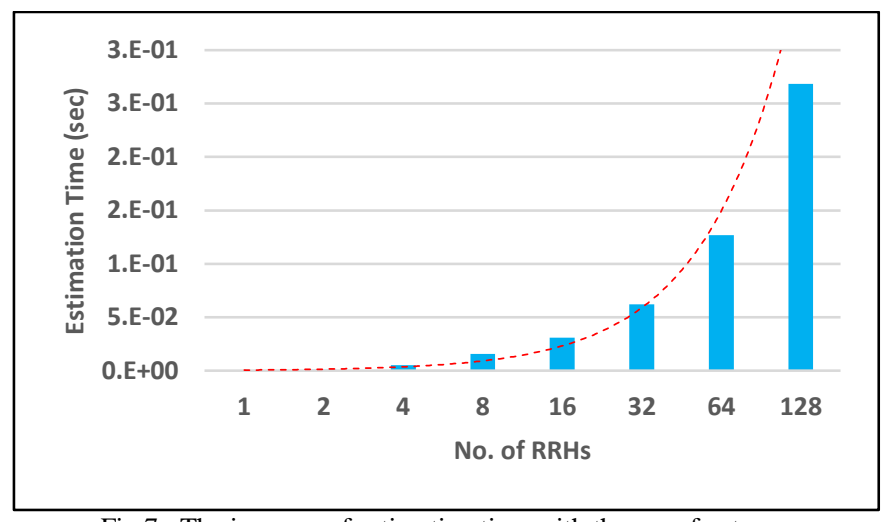

Fig.7. The increase of estimation time with the no. of antennas

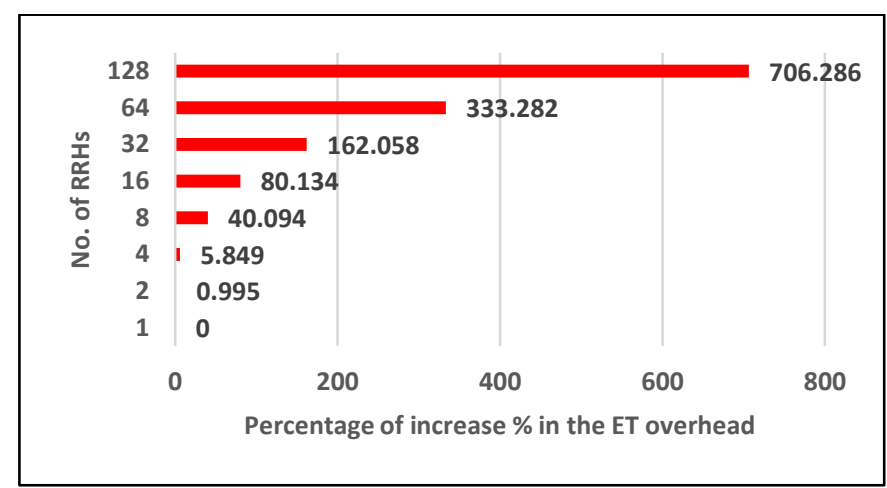

Fig. 8. Percentage of latency increase with respect to $1 \mathrm{x} 1$ antenna system

\section{B. Comparison between the complexity of MMSE and LS estimators}

The computational complexity of the channel estimation algorithms is one of the important factors that must be taken into consideration in communication systems. This is due to the fact that the time of acquisition for the channel information must be within the coherence time [32].

MMSE combines both high performance and high computational complexity simultaneously. It has a cubic computational complexity $\mathrm{O}\left(\mathrm{Y}^{3}\right)[38]$, where $\mathrm{Y}=\mathrm{N}_{\mathrm{R}} \mathrm{N}_{\mathrm{T}}$, where, in C-RAN NR $>>N T, N R$ is the number of received antennas/RRHs and NT is the number of transmitting antennas [21]. LS, on the other hand, has lower complexity $\mathrm{O}\left(\mathrm{Y}^{2}\right)$, with lower system performance in terms of data throughput at low SNRs.

This section includes an evaluation of the computational complexity of the MMSE estimator with the C-RAN architecture using 'Big-O' analysis. Big-O was used in measuring the performance of algorithms in computer science, and in this context, the complexity of the MMSE and LS algorithms. The total number of operations of the MMSE and LS estimators is illustrated in Table 1 , where the applied $\mathrm{N}$ equal to $64[35,39]$. Fig. 9 shows the computational complexity for LS and MMSE estimators in terms of number of operations. The result illustrates that the number of operations of the MMSE is $90 \%$ higher than the operations of the LS estimators. This result is compatible with the result of the previous section, which demonstrates a high overhead in the time of acquiring the channel information with the MMSE estimator, as shown recently in Fig.5.

TABLE 1: MMSE COMPUTATIONAL COMPLEXITY [35]

\begin{tabular}{|c|c|}
\hline $\begin{array}{l}\text { The component of MMSE } \\
\text { estimator for equation (3), }(\mathrm{N} \text { is } \\
\text { size of matrix) }\end{array}$ & No. of required operations \\
\hline $\mathrm{F}$ & $\mathrm{O}(\mathrm{F})=\mathrm{O}(\mathrm{DFT})=\mathrm{N}(\log \mathrm{N})$ \\
\hline Rgy & $\mathrm{O}(\mathrm{Rgy})=\mathrm{O}\left(\operatorname{Rgg~F}^{\mathrm{H}} \mathrm{X}^{\mathrm{H}}\right)=\mathrm{N}^{3}+\mathrm{N}^{2}$ \\
\hline Ryy & 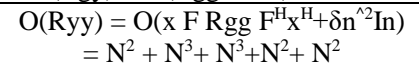 \\
\hline $\mathrm{g}_{\mathrm{mmse}}$ & 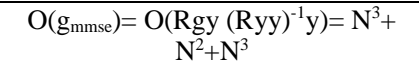 \\
\hline $\mathrm{H}_{\text {mmse }}$ & $\begin{array}{c}\mathrm{O}\left(\mathrm{h}_{\text {MMSE }}\right)=\mathrm{O}\left(\mathrm{DFT}\left(\mathrm{g}_{\text {MMSE }}\right)\right)= \\
\mathrm{N}(\log \mathrm{N})\end{array}$ \\
\hline 'For' loop & $\mathrm{O}\left({ }^{\prime}\right.$ For' loop' $)=\mathrm{N}+1, \quad$ for one loop \\
\hline $\begin{array}{l}\text { The component of LS estimator } \\
\text { for equation (2), ( } \mathrm{N} \text { is size of } \\
\text { matrix) }\end{array}$ & No. of required operations \\
\hline $\mathrm{X}^{-1}$ & $\mathrm{O}\left(\mathrm{X}^{-1}\right)=\mathrm{N}$ \\
\hline $\mathrm{X}^{-1} \mathrm{Y}$ & $\mathrm{O}\left(\mathrm{X}^{-1} \mathrm{Y}\right)=\mathrm{N}+\mathrm{N}^{2}$ \\
\hline
\end{tabular}

\begin{tabular}{|c|c|c|c|}
\hline \multirow{10}{*}{ 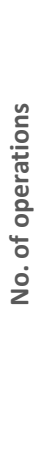 } & $1.00 \mathrm{E}+07$ & & \\
\hline & $1.00 \mathrm{E}+06$ & & \\
\hline & $1.00 \mathrm{E}+05$ & & \\
\hline & $1.00 E+04$ & & \\
\hline & $1.00 \mathrm{E}+03$ & & \\
\hline & $1.00 \mathrm{E}+02$ & & \\
\hline & $1.00 \mathrm{E}+01$ & & \\
\hline & $1.00 \mathrm{E}+00$ & & \\
\hline & & LS & MMSE \\
\hline & & Channel estimators & \\
\hline
\end{tabular}

Fig. 9. Comparison between the complexity of MMSE and LS in terms of number of operations

The former analysis has been conducted based on the architecture of the $4 \mathrm{G}$ network. In C-RAN, the major difference that should be taken into account between the distributed $4 \mathrm{G}$ and C-RAN is that in $4 \mathrm{G}$ the base band processing is achieved independently at each base station, while in C-RAN all the base band processing is aggregated at the centralised pool of VBSs in the cloud. Furthermore, the current 4G LTE-A supports up to 8 antennas, and up to 16 antennas in the new release of the Third Generation Partnership Project (3GPP), while the target number of the RRHs that C-RAN should be from 100 to 1000 per VBS $[3,4,8,14,40,41]$ to cope with the future high capacity requirements for mobile networks.

\section{SNR in $5 G$ Dense Networks}

In the next generation of mobile networks, the UEs will have higher SNR values. One of the key factors for increasing the SNR is the deployment of dense small cells. Ultra-Dense Network (UDN) is the potential theme of 5G network deployment. This theme fits well with the adoption of millimetre wave bands that require a small cell radius. The millimetre frequencies are beneficial by supporting high network capacity due to the availability of wide bandwidths [42]. However, due to the high path loss which results from its small wavelengths, there is a need to use UDN, in terms of the higher number of RRH, to compensate for the shortage of path loss in millimetre wave [43]. 
Hence, when deploying a large number of Access Points (APs), the distance between the UEs and the AP will be smaller. This structure can be considered as an advantageous point in increasing the received power by the UEs, which eventually increases their SNRs. This statement is supported by the following theoretical explanation:

According to the equation of path loss as expressed in (4) [44] it illustrates that when the distance between the UE and the RRHs deceases, the path loss decreases accordingly.

$$
L_{p}=32.4+20 \log _{10} f+20 \log _{10} d
$$

Where, $L_{p}$ is the pathloss in $\mathrm{dB}, \mathrm{f}$ is the frequency in $\mathrm{GHz}$, and $\mathrm{d}$ is the distance between the base station and the user in metres. Therefore, as the distance between the antennas of the transmitter and the receiver increases, the path loss increases accordingly. This is also applied to the carrier frequency. Therefore, future networks in millimetre waves will suffer high path loss.

Friis formula is a mathematical expression used to calculate the received power based on specific transmission parameters. The formula in general states that the received signal power will increase when the path loss decreases, as shown below [45].

$P_{r}=P_{T}+G_{T X}+G_{R X}-L_{p}$

Where, $P_{r}$ is the power of the received signal, $\mathrm{P}_{\mathrm{T}}$ is the transmission power. $G_{T X}$ and $G_{R X}$ are the gain of transmitting and receiving antennas respectively and $L_{p}$ is the path loss signal as in Eq.(4).

Based on the received power in equation (5), the UE SNR will be calculated by dividing the power of the received signal on the noise power as follows [46, 47]:

SNR $=\frac{\text { Signal power }}{\text { Noise power }}$

According to equation 4 , it is noticed that in the case of decreasing the distance between the AP and the UEs, the path loss in Eq.4 will also be decreased accordingly. Eventually the SNR will increase when increasing the number of the RRHs.

To clarify this, we conducted a numerical test to see the relation between the path loss and distance $\mathrm{d}$ with the following parameter settings: $P_{T}$ is $40 \mathrm{~dB}, \mathrm{G}_{\mathrm{TX}}$ and $\mathrm{G}_{\mathrm{RX}}$ are 15 and zero $\mathrm{dB}$ respectively, noise power is $-101 \mathrm{~dB}$, the distance range from 100 to 1000 meter and $28 \mathrm{GHz}$ carrier frequency. As shown in Fig.10, the path loss will be decreased significantly as the inter site distance is decreased. Therefore, a wireless network with high RRH density will significantly improve the SNR. The results demonstrate that when the distance increases, the SNR reduces accordingly, which is also shown in Fig.10 as a result of closer access point to the users, which improves the received signal power.

It is worth mentioning that for the sake of simplicity in the former analysis, the interference has been neglected, yet even if the interference was taken into consideration, the values of SNRs still increase by shortening the distance between the antennas of the UE and the base station.
As a result of likely increased SNR regions in the $5 \mathrm{G}$ era, a more efficient algorithm must be used to take advantage of increased SNR. Therefore the LS estimator, which has almost similar performance to MMSE at high SNRs, can be used to gain latency reduction. LS has superior performance in terms of latency compared to MMSE, however it has poor performance in low SNR regions.

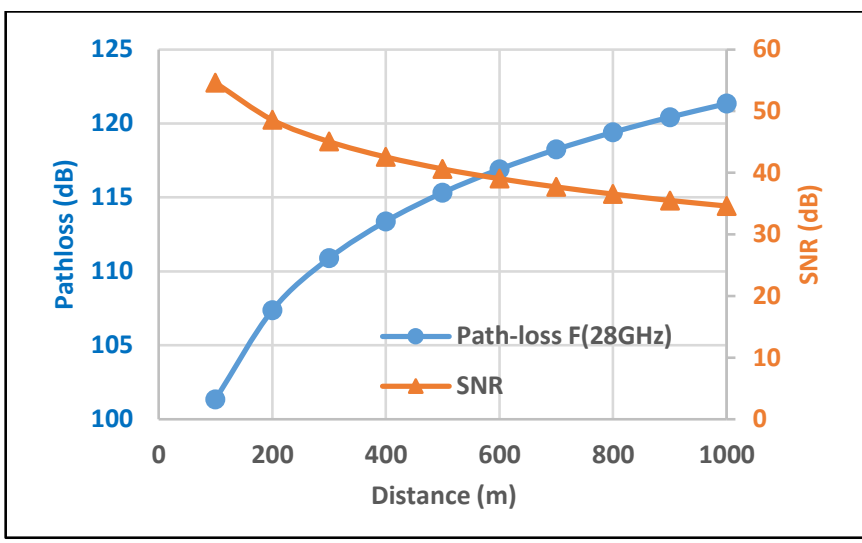

Fig.10. Path loss and SNR vs distance

\section{DyNAMiC CHANNEL ESTIMATORS SELECTION ALGORITHM}

In this section, the Dynamic Channel Estimator Selection Algorithm (DCESA) is presented to reduce the estimation time of CSI acquisition. In this algorithm, the MMSE has been used below SNR threshold to maximise or improve throughput and the LS estimator above the SNR threshold to minimise latency. In other words, this technique is used to assign dynamically the users that have high SNR, to one estimator (e.g. the LS) while the low SNR UEs are assigned to another estimation algorithm (e.g. MMSE estimator). Throughout this study, two estimation algorithms are used as a case study scenario: MMSE for low SNR and LS for high SNR. Deploying a hybrid channel estimation algorithm can be achieved according to the following.

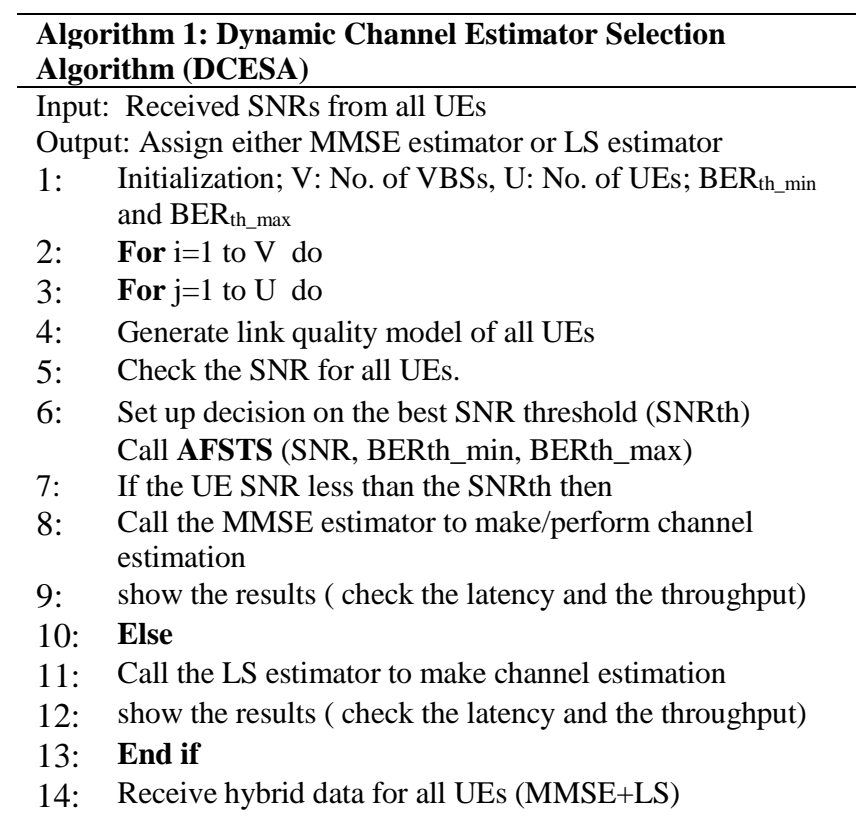


15: Compare the results and evaluate the overall gain in estimation time and the loss in the throughput

16: End for

17: End for

18: End

\section{Adaptive SNR threshold Selection using Fuzzy logic}

The value of the chosen SNR threshold $\left(\mathrm{SNR}_{\mathrm{th}}\right)$ has a direct effect on the outcome of the proposed DCESA, in terms of the ET gain and the TP loss. For the purpose of automatic selection to the value of this threshold, the concept of artificial intelligence 'fuzzy logic' is discussed in the next sub section.

\section{a) Fuzzy Logic Threshold Selection}

The challenge of allocating a suitable $\mathrm{SNR}_{\mathrm{th}}$ is in the balance between the gain estimation time and the loss of the data throughput. Hence, the intended objective is to choose a threshold that satisfies the highest gain in ET and lowest loss in data throughput. To achieve this trade-off, Fuzzy Logic (FL) is employed to select the best $\mathrm{SNR}_{\text {th. }}$. In this work, the criteria for performance measurement of choosing the SNR threshold for the purpose of using a mixed channel estimator algorithm depends on the value of the received SNR and the corresponding Bite Error Rate (BER). This is due to the fact that the SNR represents the quality of the signal, while the BER shows the error rate in signal. In fact, the relation between the BER and the SNR is an inverse relation; when the SNR increases, the BER decreases proportionally and vice versa. It is worth stating that FL has been proposed in this study due to many considerations, which include the application being able to cover a much broader range of operating conditions with the presence of uncertainty in measurements. Secondly, FL is customisable, since it is easier to understand and modify rule sets, which not only includes the consideration of human operators, but also is expressed in natural linguistic terms. Another consideration is that FL can be applied on-line without a learning phase. Finally, the combination of the two input variables, the error and the change in error (which are represented by the BER and the $\triangle B E R$ in this study) have been used as inputs to FL in other fields of knowledge to predict the behaviour of systems and then to produce the best decision for the desired output variables, such as in control system applications [48]. For all of the above considerations, FL was chosen in this study.

The structure of an FL algorithm is illustrated in Fig.11 and a brief explanation for the fuzzy components is shown as follows: 1) There are two input variables for the FL algorithm, namely BER (and change in BER), while the output is the best SNR threshold.

2) Five uniform triangular membership functions (as depicted in Fig.12) have been applied with an overlap of 50\% for the input variables. While in the output of FL, seven singleton membership functions have been used.

3) The Mamdani set of fuzzy rules is applied here as shown in Table 2. The general Mamdani fuzzy rule for Multiple Inputs Single Output (MISO) algorithm is:
(IF $x_{l}$ is $\tilde{A} \tilde{i}$ AND ... AND $x_{M}$ is $\tilde{A}{ }_{M}$ THEN $z$ is $\tilde{B}$ ).

4) The most common technique, the Centroid, also known as the Centre of Gravity method (COG), is used as a defuzzification method.

5) The rules of FL are derived based on the idea in Fig.13. This concept has been applied in control systems to attain the desired response [48, 49]. The details of the applied procedure for selecting the optimum $\mathrm{SNR}_{\text {th }}$ using $\mathrm{FL}$ is illustrated in Algorithm 2.

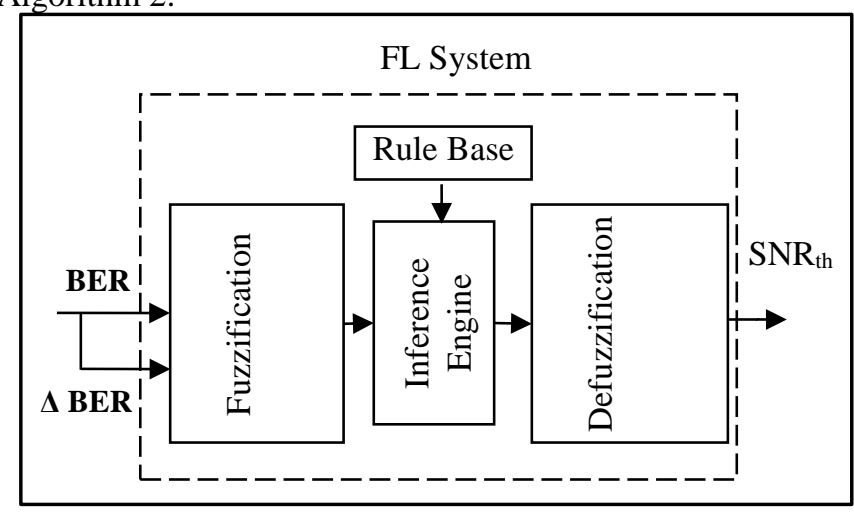

Fig.11. Structure of fuzzy System

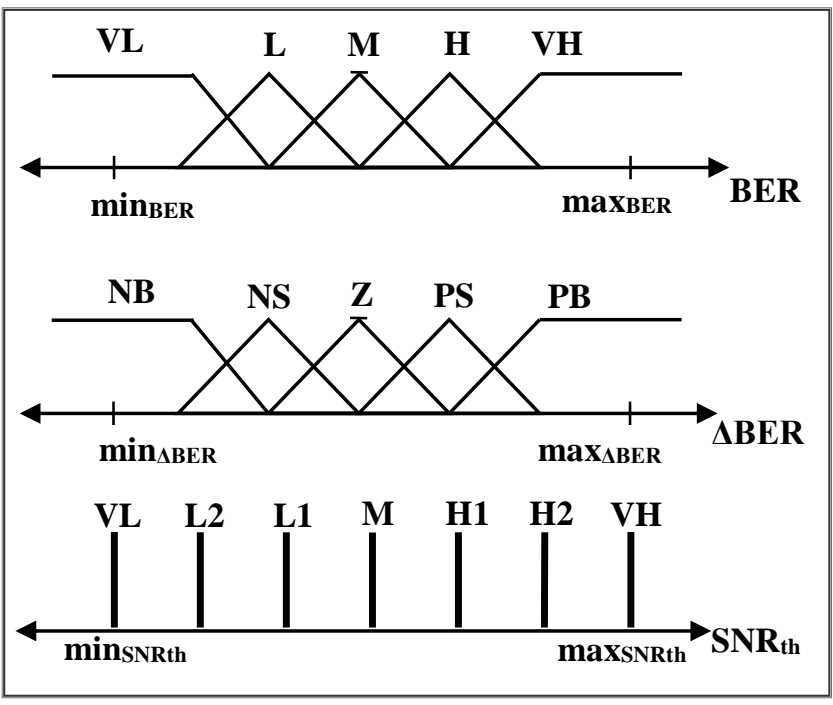

Fig.12. Input and output memberships of FL

\begin{tabular}{|c|c|c|c|c|c|c|}
\hline \multicolumn{2}{|c|}{ SNR } & \multicolumn{5}{c|}{ BABLE 2: FUZZY RULE BASE } \\
\cline { 2 - 7 } & VL & L & M & H & VH \\
\hline \multirow{5}{*}{$\Delta$ BEER } & NB & VL & VL & L2 & L1 & M \\
\cline { 2 - 7 } & NS & VL & L2 & L2 & M & H1 \\
\cline { 2 - 7 } & L2 & L2 & M & H2 & H2 \\
\cline { 2 - 7 } & PS & L1 & M & H2 & H2 & VH \\
\cline { 2 - 7 } & PB & M & H1 & H2 & VH & VH \\
\hline
\end{tabular}




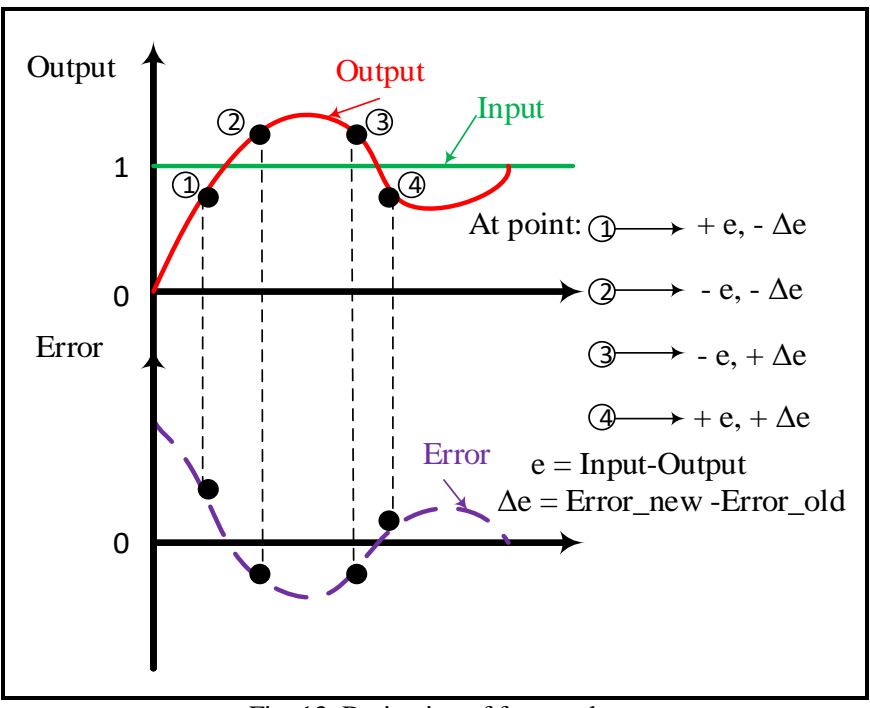

Fig. 13. Derivation of fuzzy rules

\section{Algorithm 2: Adaptive Fuzzy SNR Threshold Selection (AFSTS)}

Input:

Received SNR

Received two BER thresholds

Output:

Best SNR threshold $\left(\mathrm{SNR}_{\mathrm{th}}\right)$

1: Determine the value of the BER from the received SNR of each UE.

2: Calculate the value of change in the bit error rate $(\triangle B E R)$ (BERnew-BERold), for the purpose of predicting whether the new BER will increase or decrease proportionately to the value of the SNR.

3: Assign the inputs to FL (BER \& $\triangle \mathrm{BER}$ )

4: The fuzzy inference system will choose the best $S_{N R}$ th based on the values of inputs.

5: When the value of BER is equal or larger than the minimum BER threshold (BERth_min), FL will start to select the lower SNRth threshold, which means lower usage of the MMSE than LS estimator, as shown in Fig.14 to increase the ET gain.

6: When the value of BER is equal or larger than the maximum BER threshold (BERth_max), FL starts to assign the higher threshold SNRth, where the higher SNRth means the usage of MMSE estimator is higher than the LS, to minimize the TP loss, as shown in Fig. 14.

7: $\quad$ Return the selected SNRth.

8: End

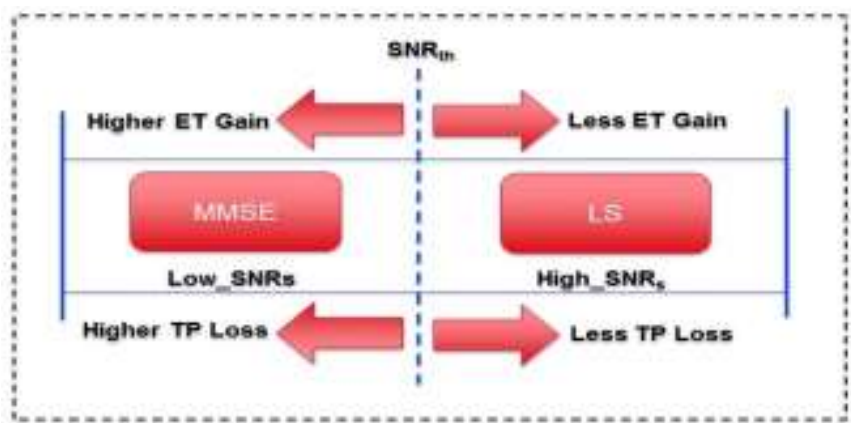

Fig. 14. SNR threshold vs. the change in ET (increase or decrease) using the mixed estimators approach

\section{b) Calculation of Bit Error Rate in OFDM System}

BER is a key metric to measure the quality of a wireless communication channel and then to evaluate the performance of the communication system. A higher BER indicates a slower data rate. In fact, the BER has a direct relation with the value of the SNR [50]. Hence, in this work, the BER and $\triangle \mathrm{BER}$ have been considered to quantify the performance of the communication link from the SNR values. Increasing the SNR means a higher link quality and low BER, while low SNR values indicate an increase in the BER and a poor communication link. The general definition of the BER is the average rate of bits error divided by the total transmitted bits. However, since this research investigates the correlation between the BER and the SNR, the value of BER can be calculated in terms of SNR. For the purpose of analysis, Quadrature Amplitude Modulation (QAM) has been used due to its simplicity in implementation. Nonetheless, the work can be extended to other modulation techniques by changing the formulae according to the applied technique. Therefore, the performance of BER for the QAM system can be expressed in the following equations [51, 52].

$$
\begin{aligned}
& B E R=\frac{4}{C} \cdot\left(1-\frac{1}{2^{\frac{C}{2}}}\right) \cdot Q\left(\sqrt{3 \cdot \frac{S N R}{2^{C}-1}}\right) \\
& S N R=\frac{P_{S}}{P_{n}} \cdot L \\
& S N R=S_{o} \alpha \cdot R_{S}
\end{aligned}
$$

where, $\boldsymbol{C}$ is the number of bits per symbol; $\boldsymbol{P}_{\boldsymbol{S}}$ is the power of transmission; $\boldsymbol{L}$ includes the entire transmission loss components; $\boldsymbol{P}_{\boldsymbol{n}}$ is the noise power; $\boldsymbol{S}_{\boldsymbol{o}}$ is noise power spectral density; $\boldsymbol{\alpha}$ is a factor that takes into account all other elements, e,g. filter non-idealities.

\section{PERFORMANCE EVALUATION AND SimUlation RESUltS}

Based on the results shown above, the MMSE has good system performance in terms of data throughput, but with a higher number of operations and estimation time. Also, this system has a complexity $\mathrm{O}\left(\mathrm{Y}^{3}\right)$, which yields higher latency. In contrary, the LS estimator has lower performance at low SNRs in terms of data throughput and an almost identical performance as MMSE at high SNR, but with a lower number of operations and estimation time, and has less complexity of $\mathrm{O}\left(\mathrm{Y}^{2}\right)$, which yields low latency. Therefore, the proposed algorithm will harness the MMSE at low SNRs region to gain higher data TP, while exploiting the LS algorithm at high SNRs region to gain lower complexity and hence lower end to end latency. The algorithm then will aggregate the overall outcome. A threshold for SNR selection has been considered for the proposed algorithms to balance between LS and MMSE algorithm. However, as shown in Section (III, C) with the future structure of mobile networks, most UEs will have high SNR figures. Thus, the gain of reduction in the latency will be higher if the UE has high SNR or equivalently the $\mathrm{SNR}_{\text {th }}$ will be larger and the usage of LS will be higher than the MMSE. The expected gain in latency and loss in throughput that can be achieved in the proposed algorithm according to the value of the selected $S_{N R}$ are clarified in Fig.14. The results of the study are obtained using MATLAB R2016a. The applied parameter settings are depicted in Table 3. At the start, the applied SNR threshold was chosen manually to illustrate the impact of the proposed DCESA 
throughout different SNR thresholds on the network performance. Then, the automatic selection for $\mathrm{SNR}_{\text {th }}$ using FL is employed and tested for different SNR ranges, as shown in the second part of the simulation results.

TABLE 3: SySTEM MODEL PARAMETERS

\begin{tabular}{|c|c|}
\hline Parameters & Values \\
\hline $\begin{array}{c}\text { SNR threshold (for manual } \\
\text { selection) }\end{array}$ & $10,15,20(\mathrm{~dB})$ \\
\hline Antenna system & $2 \times 2,4 \times 16$ \\
\hline Carrier frequency & $1.9 \mathrm{GHz}$ \\
\hline System bandwidth & $1.4 \mathrm{MHz}$ \\
\hline Transmission mode & CLSM \\
\hline Multiplexing & OFDM \\
\hline Channel estimator & LS, MMSE, Proposed DCESA \\
\hline Network size & Single VBS, 5UEs \\
\hline UE mobility speed & $100 \mathrm{~km} / \mathrm{hr}$ \\
\hline BER range & $0-10^{-6}$ \\
\hline
\end{tabular}

For manual $\mathrm{SNR}_{\text {th }}$ selection tests, to quantify the saving in ET, three tests were conducted based on three SNR thresholds and two antenna systems (as shown in Table 3) for each test. At each simulation test, four types of sub figures are plotted to evaluate the performance of the DCESA. The chosen SNR threshold maximises TP and minimises latency. Three SNR thresholds are applied to show the benefits of using mixed channel estimation algorithms. Generally, the results in the three tests are shown in Fig. 15 to Fig. 19, where, the performance metrics used are: 1) ET gain which represents the gain percentage of reduction in the ET using DCESA compared to the ET of the MMSE. 2) TP loss which represents the loss percentage in data TP with the DCESA compared to the MMSE data TP. For a $10 \mathrm{~dB}$ SNR threshold, Fig. 15 reveals that the proposed algorithm gain in ET is significant, while the loss in the overall cell throughput is trivial. This is for a $2 \times 2$ MIMO channel. For $15 \mathrm{~dB}$ SNR threshold, Fig. 16 shows that with 15 $\mathrm{dB}$ SNR the ET gain has been noticeably decreased with a reduction to the data throughput losses, In the $20 \mathrm{~dB}$ SNR threshold, the results in Fig.17 for $2 \times 2$ antenna system) show low ET gain and less loss in the TP.

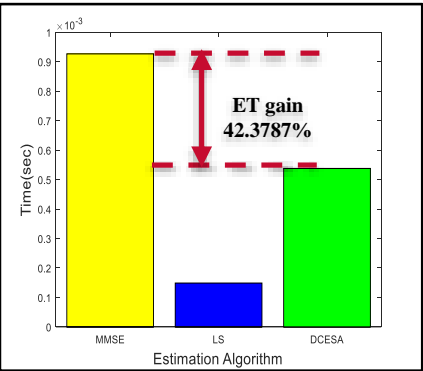

a) ET

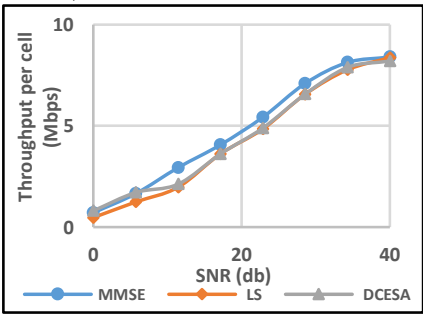

c) Cell TP

Fig.15. Mixed Estimators with (2x2 antenna system and 10dB SNR)

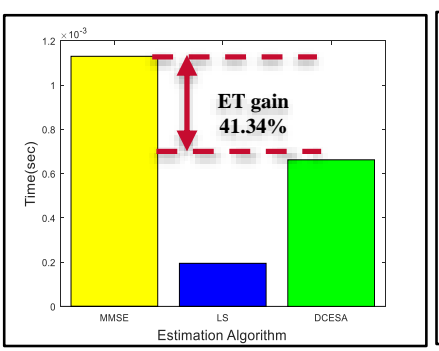

a) ET

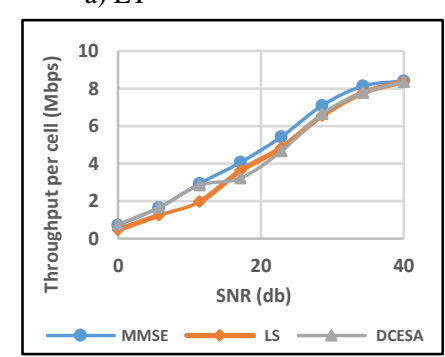

c) Cell TP

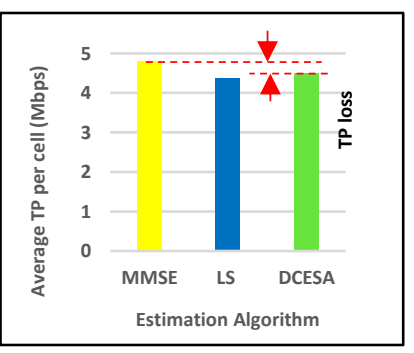

b) Average Cell TP

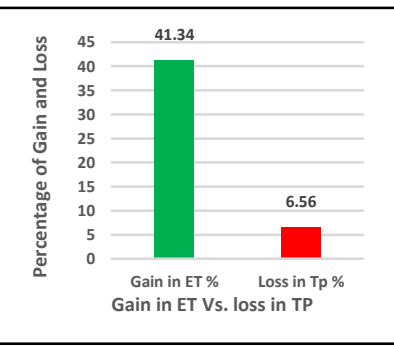

d) Gain in ET Vs. Loss in TP

Fig.16. Mixed Estimators with (2x2 antenna system and 15dB SNR)

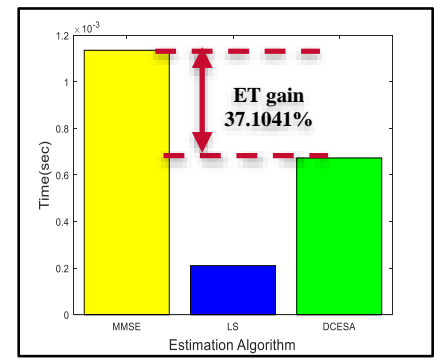

a) ET

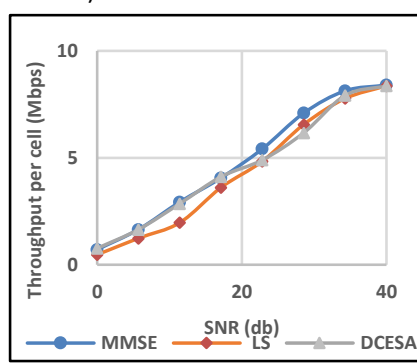

c) Cell TP

Fig.17. Mixec

Now, higher numbers of RRHs have been considered in this work to demonstrate and validate our findings for the $2 \times 2$ antenna system. A 4x16 MIMO system has been used, as the CRAN network can support easier scalability for adding more RRHs. The results in Table 4 demonstrate that much higher performance gain can be achieved when using the proposed DCESA compared to a $2 \times 2$ system.

It is worth stating that the MMSE has been considered as the performance reference throughout all comparisons in this work. A noticeable point can be seen in the results of the $2 \times 2$ system (Figures 15 to 17 ) and $4 \times 16$ system (Table 4 ), which is when the SNR threshold starts to increase, the percentage gain in ET decreases and the TP loss also decreases. The explanation is clarified in Fig. 14, where larger SNR values mean higher usage to the MMSE compared to the LS estimator. 
TABLE 4: PERFORMANCE OF 4X16 ANTENNA SYSTEM USING MIXED ESTIMATION ALGORITHM

\begin{tabular}{|c|c|c|c|}
\hline $\begin{array}{c}\text { Performance } \\
\text { metrics } \\
\text { ET\&TP }\end{array}$ & $\begin{array}{c}\text { SNR }_{\text {th }} \\
\mathrm{dB} 10\end{array}$ & $\begin{array}{c}\mathrm{SNR}_{\text {th }} \\
\mathrm{dB} 15\end{array}$ & $\begin{array}{c}\mathrm{SNR}_{\text {th }} \\
\mathrm{dB} 20\end{array}$ \\
\hline \multicolumn{4}{|c|}{ ET (sec) } \\
\hline MMSE & 0.021 & 0.019 & 0.018 \\
\hline LS & 0.001 & 0.00095 & 0.00097 \\
\hline DCESA & 0.0107 & 0.0101 & 0.0098 \\
\hline ET gain $(\%)$ & 48.650 & 47.365 & 45.455 \\
\hline \multicolumn{4}{|c|}{ TP $(\mathrm{Mbps})$} \\
\hline MMSE & 13.069 & 13.068 & 13.069 \\
\hline LS & 12.1904 & 12.0654 & 12.0653 \\
\hline DCESA & 12.627 & 12.7691 & 13.0584 \\
\hline TP loss $(\%)$ & 3.382 & 2.2947 & 0.0811 \\
\hline
\end{tabular}

Furthermore, regarding the value of the SNR threshold, the results in Fig.18 (which are the summary of all previous tests) demonstrate that for a higher SNR threshold 15 and $20 \mathrm{~dB}$, the overall gain in ET starts to decrease since the low overhead LS estimator is used after these SNR thresholds. At the same time, lower loss in the overall data throughput is noticed with the high SNR thresholds, as shown in Fig. 19 or equivalently the higher TP gain is observed as a result of using the MMSE estimator below these high SNR thresholds. Hence, this means that there is a trade-off between the chosen SNR thresholds, the system TP and the ET. Therefore, for real implementation, it is beneficial for the SNR threshold to be adaptive based on the link quality of the UEs.

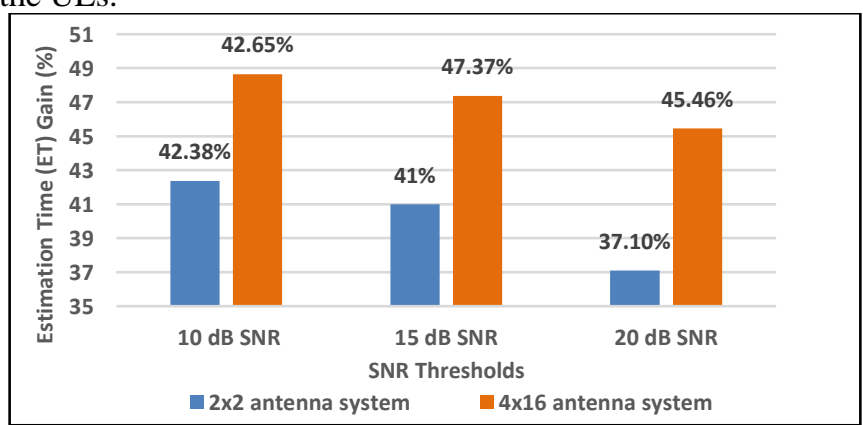

Fig.18. Comparison between the SNR thresholds (manually selected) in terms of ET gain, compared to MMSE

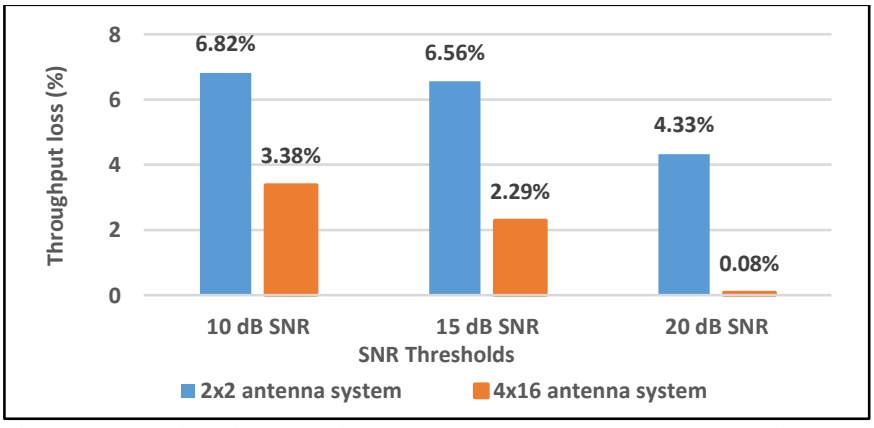

Fig.19. Comparison between the SNR thresholds (manually selected) in terms of TP loss, compared to MMSE
The second part of the simulation results focuses on deploying adaptive SNR thresholds using FL to maintain the trade-off balance between the gain in ET and the loss in TP. The first advantage of using adaptive $\mathrm{SNR}_{\mathrm{th}}$ is to select the value of the threshold automatically based on the value of BER and $\triangle B E R$. Hence, more accurate results are achieved with the application of the automatic SNR threshold selection using FL in each subframe. Again, a 2x2 MIMO system is used for the purpose of comparison, where the $\mathrm{SNR}_{\mathrm{th}}$ is adaptive. The result in Table 5 is produced with the existence of FL for automatic SNR $_{\text {th }}$ selection, in this result, the proposed DCESA still maintains high ET gain and low TP loss similar to manual SNR selection. However, this results is considered a more optimised as the threshold has been chosen based on FL for each subframe. Also, this is a validation for the previous results, but now the threshold is automatically selected. Likewise, for the purpose of fair comparison, a test has been conducted to show the benefits of using FL for $4 \times 16$ antenna system. The result still maintains higher gain in ET and lower loss in TP at higher number of antennas as shown in Table 5.

TABLE 5: COMPARISON THE PERFORMANCE OF 2X2 AND 4X16 ANTENNA SYSTEMS USING FL FOR AUTOMATIC SNR THRESHOLD SELECTION

\begin{tabular}{|c|c|c|}
\hline $\begin{array}{c}\text { Performance } \\
\text { metrics } \\
\text { ET\&TP }\end{array}$ & $\begin{array}{c}2 \times 2 \\
\text { antenna } \\
\text { system }\end{array}$ & $\begin{array}{c}4 \times 16 \\
\text { antenna } \\
\text { system }\end{array}$ \\
\hline \multicolumn{3}{|c|}{ ET (sec) } \\
\hline MMSE & 0.001 & 0.023 \\
\hline LS & 0.00019 & 0.00125 \\
\hline DCESA & 0.00058 & 0.0121 \\
\hline ET gain $(\%)$ & 40.17 & 47.35 \\
\hline \multicolumn{2}{|c|}{ TP $(\mathrm{Mbps})$} \\
\hline MMSE & 4.8015 & 13.02 \\
\hline LS & 4.3552 & 12.0461 \\
\hline DCESA & 4.693 & 12.887 \\
\hline TP loss $(\%)$ & 2.26 & 1.02 \\
\hline
\end{tabular}

Fig.20 shows the ET gain for different ranges of the overall applied SNRs. The result demonstrates almost similar values in the percentage of ET gain. This is due to the optimal decision of FL for choosing the value of the SNR threshold. The results in Fig. 21 show that when the range of SNR starts to increase, the loss in the data throughput reduces proportionately. This is due to the fact that the threshold can now shift to longer SNR, allowing more room for MMSE operation, which improves the TP. Therefore, the percentage of loss in TP decreased as the SNR threshold increase. The results in Fig.22 demonstrate a noticeable difference in the MSE, where at the low SNRs the LS shows high MSE and the error start to decrease at high SNR values, which is the reason behind using the algorithm of mixed estimators. On the other hand, the MMSE estimator and our proposed algorithm show lower SNRs in all ranges. 


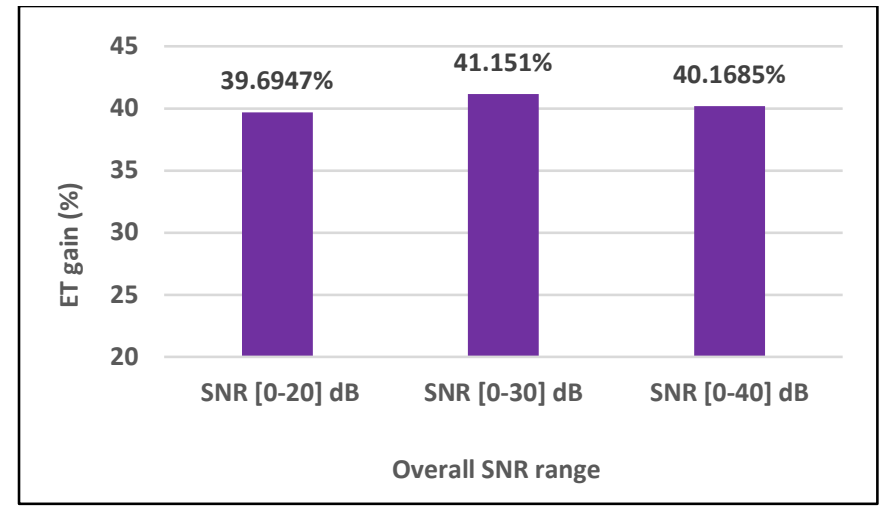

Fig.20. ET gain for different SNR ranges with automatic $\mathrm{SNR}_{\text {th }}$ selection for $2 \times 2$ system

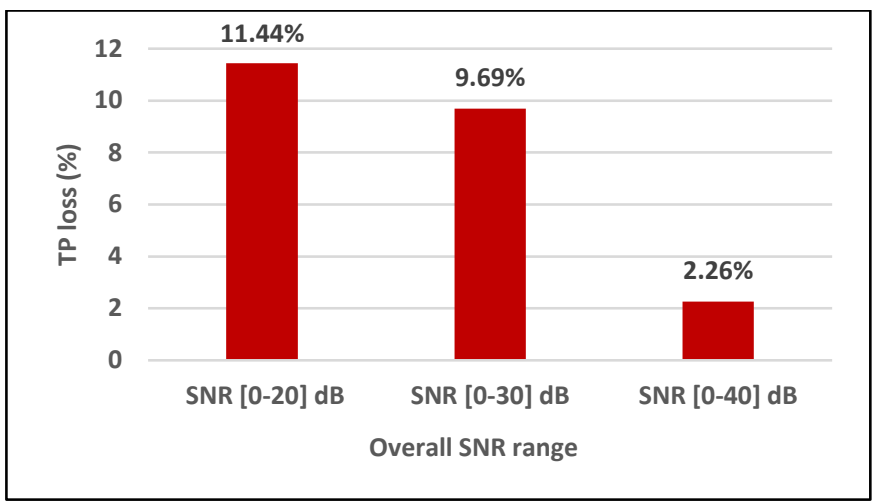

Fig.21. TP loss for different SNR ranges with automatic $\mathrm{SNR}_{\mathrm{th}}$ selection for $2 \times 2$ system

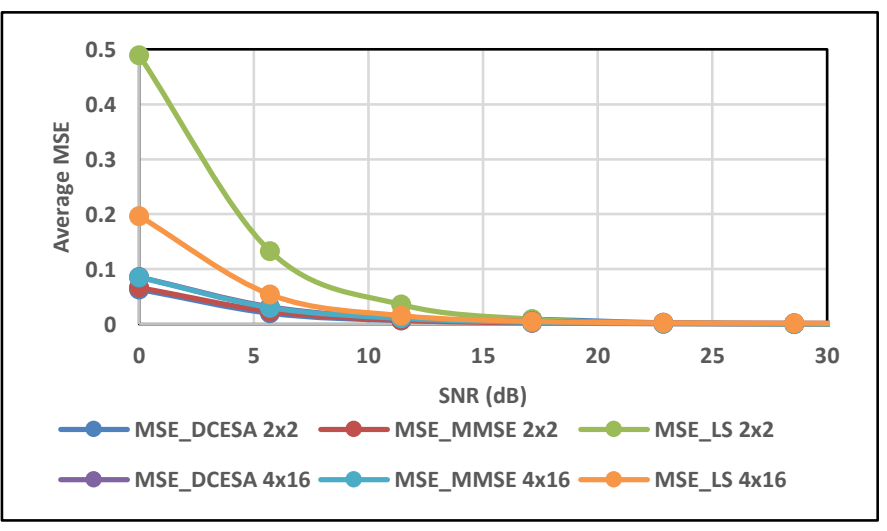

Fig. 22. Composition between the performance of the DCESA, MMSE and LS in terms of MSE

\section{CONCLUSIONS AND RECOMMENDATIONS}

$\mathrm{C}-\mathrm{RAN}$ is one of the most promising technologies to meet the demand of the future cellular networks. The overhead of the CSI acquisition in dense C-RAN networks is one of the key challenges for deploying C-RAN in an efficient manner. In this paper, the DCESA is employed to reduce the estimation time overhead and maintain the performance of the network. The obtained results demonstrate that DCESA has the potential to save a significant amount of estimation time in CSI acquisition. This gain has been achieved by utilising the desired merits of both the MMSE and the LS estimators, while keeping the performance of the network without noticeable degradation. The reduction in estimation time will contribute to minimising the overall end to end latency in C-RAN networks. However, all the previous tests were for relatively modest antenna numbers and the results show an obvious gain in the latency, so it will be beneficial to adopt the DCESA for networks accommodating a large number of antennas (100-1000).

For the purpose of deploying adaptive SNR thresholds to satisfy an optimal trade-off between the gain in the data throughput and latency in CSI acquisition, the concept of FL has been utilised by considering the BER as a function of SNR to capture the performance wireless communication channel. As a secondary advantage of implementing our algorithm, a reduction in the interference could be achieved as a result of better CSI acquisition. Therefore, we could compare the improvement of this technique with the standard schemes of CoMP, and this will be the subject of our future work.

In addition, due to the negligible throughput losses, it is recommended to use this algorithm in future wireless networks in order to provide lower end to end latency to satisfy UE demands and to fulfil services that require shorter latency, such as the Internet of Things (IoT), Vehicle to Vehicle V2V communication, disaster resilience applications and health care emergency.

\section{ACKNOWLEDGMENT}

This research has been funded by the Ministry of Higher Education and the Scientific Research of Iraq - University of Technology.

\section{REFERENCES}

G. Liu and D. Jiang, "5G: Vision and Requirements for Mobile Communication System towards Year 2020," Chinese Journal of Engineering, vol. 2016, 2016.

M. Peng, Y. Li, Z. Zhao, and C. Wang, "System architecture and key technologies for $5 \mathrm{G}$ heterogeneous cloud radio access networks," IEEE Network, vol. 29, pp. 6-14, 2015.

Mobile China, "C-RAN: the road towards green RAN," White Paper, ver, vol. 2, 2011.

A. Checko, H. L. Christiansen, Y. Yan, L. Scolari, G. Kardaras, M. S. Berger, et al., "Cloud RAN for mobile networks - a technology overview," Communications Surveys \& Tutorials, IEEE, vol. 17, pp. 405-426, 2015.

Y. Shi, J. Zhang, and K. Letaief, "CSI overhead reduction with stochastic beamforming for cloud radio access networks," in IEEE International Conference on Communications (ICC) 2014, pp. 5154-5159.

C. Fan, Y. J. A. Zhang, and X. Yuan, "Scalable coordinated uplink processing in cloud radio access networks," in IEEE Global Communications Conference (GLOBECOM), 2014, pp. 3591-3596. H. Zarrinkoub, Understanding LTE with MATLAB: From Mathematical Modeling to Simulation and Prototyping: John Wiley \& Sons, 2014.

Y. Shi, J. Zhang, K. B. Letaief, B. Bai, and W. Chen, "Large-scale convex optimization for ultra-dense Cloud-RAN," Wireless Communications, IEEE, vol. 22, pp. 84-91, 2015.

Y. Shi, J. Zhang, and K. Letaief, "Optimal stochastic coordinated beamforming with compressive CSI acquisition for Cloud-RAN," arXiv preprint arXiv, vol. 1312, 2013.

A. Liu and V. K. Lau, "Joint power and antenna selection optimization in large cloud radio access networks," Signal Processing, IEEE Transactions on, vol. 62, pp. 1319-1328, 2014. 
[11] K. Ntougias, D. Ntaikos, and C. B. Papadias. (2015). Reducing Complexity in Next-Generation MU-MIMO Systems. Available: https://arxiv.org/abs/1507.04050

[12] S. Roy, "Two-Layer Linear Processing for Massive MIMO on the TitanMIMO Platform," 2015.

[13] A. M. Mahmood and A. Al-Yasiri, "Scalable Processing in 5G Cloud-RAN Networks Using MapReduce Framework," in New Technologies, Mobility and Security (NTMS), 2016 8th IFIP International Conference on, 2016, pp. 1-6.

[14] C. Fan, Y. J. Zhang, and X. Yuan, "Dynamic Nested Clustering for Parallel PHY-Layer Processing in Cloud-RANs," IEEE Transactions on Wireless Communications, vol. 15, pp. 1881-1894, 2016.

[15] D. Liu, S. Han, C. Yang, and Q. Zhang, "Semi-Dynamic UserSpecific Clustering for Downlink Cloud Radio Access Network," IEEE Transactions On Vehicular Technology, vol. 65, 2016.

[16] K. Wu and D. Li, "Channel Estimation for Large Antenna Systems," in IEEE 81st Vehicular Technology Conference (VTC Spring), 2015, pp. 1-5.

[17] L. Liu and R. Zhang, "Optimized uplink transmission in multiantenna C-RAN with spatial compression and forward," IEEE Transactions on Signal Processing, vol. 63, pp. 5083-5095, 2015.

[18] J. Lorca and L. Cucala, "Lossless compression technique for the fronthaul of LTE/LTE-advanced Cloud-RAN architectures," in IEEE 14th International Symposium and Workshops on a World of Wireless, Mobile and Multimedia Networks (WoWMoM), 2013, pp. $1-9$.

[19] Z. Zhao, M. Peng, Z. Ding, W. Wang, and H. V. Poor, "Cluster Content Caching: An Energy-Efficient Approach to Improve Quality of Service in Cloud Radio Access Networks," arXiv preprint arXiv:1603.07052, 2016

[20] Z. Mao, M. Peng, H. Wang, J. Zhou, and X. Xie, "Low-Complexity Segment Training Channel Estimation in Cloud Radio Access Networks," in Vehicular Technology Conference (VTC Fall), 2015 IEEE 82nd, 2015, pp. 1-5.

[21] N. Shariati, E. Björnson, M. Bengtsson, and M. Debbah, "Lowcomplexity polynomial channel estimation in large-scale MIMO with arbitrary statistics," IEEE Journal of Selected Topics in Signal Processing, vol. 8, pp. 815-830, 2014.

[22] A. Hajisami and D. Pompili, "Cloud-CFFR: Coordinated Fractional Frequency Reuse in Cloud Radio Access Network (C-RAN)," in Mobile Ad Hoc and Sensor Systems (MASS), 2015 IEEE 12th International Conference on, 2015, pp. 46-54.

[23] A. Hajisami and D. Pompili, "DJP: Dynamic Joint Processing for Interference Cancellation in Cloud Radio Access Networks," in Vehicular Technology Conference (VTC Fall), 2015 IEEE 82nd, 2015, pp. 1-5.

[24] A. Khlifi and R. Bouallegue, "Performance analysis of LS and LMMSE channel estimation techniques for LTE downlink systems," arXiv preprint arXiv:1111.1666, 2011.

[25] Y. Cai, F. R. Yu, and S. Bu, "Cloud computing meets mobile wireless communications in next generation cellular networks," IEEE Network, vol. 28, pp. 54-59, 2014.

[26] A. M. Mahmood, A. Al-Yasiri, and O. Y. Alani, "A New Processing Approach for Reducing Computational Complexity in Cloud-RAN Mobile Networks," IEEE Access, 2017.

[27] E. J. Kitindi, S. Fu, Y. Jia, A. Kabir, and Y. Wang, "Wireless network virtualization with SDN and C-RAN for 5G networks: Requirements, opportunities, and challenges," IEEE Access, vol. 5 , pp. 19099-19115, 2017.

[28] T. X. Tran, A. Hajisami, and D. Pompili, "Cooperative hierarchical caching in 5G cloud radio access networks," IEEE Network, vol. 31, pp. 35-41, 2017.

[29] K. Chen and R. Duan, "C-RAN: The Road Towards Green RAN, White Paper Version 2.5, China Mobile Research Institute, Oct. 2011," ed, 2011

[30] N. Alliance, "Suggestions on potential solutions to C-RAN," 2013.

[31] Y. Lin, L. Shao, Z. Zhu, Q. Wang, and R. K. Sabhikhi, "Wireless network cloud: Architecture and system requirements," IBM Journal of Research and Development, vol. 54, pp. 4: 1-4: 12, 2010. C. Cox, An introduction to LTE: LTE, LTE-advanced, SAE and $4 G$ mobile communications: John Wiley \& Sons, 2012.
Y. Cai, F. R. Yu, and S. Bu, "Dynamic operations of cloud radio access networks (C-RAN) for mobile cloud computing systems," 2015.

[34] K. N. Ntougias, Dimitrios; Papadias, Constantinos B, "Reducing Complexity in Next-Generation MU-MIMO Systems," eprint arXiv:1507.04050, 2015

[35] M. N. I. Khan and M. J. Alam, "Noise reduction algorithm for LS channel estimation in OFDM system," in Computer and Information Technology (ICCIT), 2012 15th International Conference on, 2012, pp. 310-315.

[36] Y. Shen and E. Martinez, "Channel estimation in OFDM systems," Freescale Semiconductor Application Note, pp. 1-15, 2006.

[37] S. Galih, T. Adiono, and A. Kurniawan, "Low Complexity MMSE Channel Estimation by Weight Matrix Elements Sampling for Downlink OFDMA Elements Sampling for Downlink OFDMA Mobile WiMAX Mobile WiMAX System," IJCSNS, vol. 10, p. 280, 2010.

[38] Y. L. V Savaux, "LMMSE channel estimation in OFDM context: a review," IET Signal Processing Journal, vol. 11, 2017.

[39] M. J. Alam, "Low Complexity Channel Estimation for OFDM Systems Based on LS and MMSE Estimators'," Blekinge Institute of Technology, 2010.

[40] Y. Shi, J. Zhang, and K. B. Letaief, "Optimal stochastic coordinated beamforming for wireless cooperative networks with CSI uncertainty," IEEE Transactions on Signal Processing, vol. 63, pp. 960-973, 2015

[41] H. Zhang, C. Jiang, J. Cheng, and V. C. Leung, "Cooperative interference mitigation and handover management for heterogeneous cloud small cell networks," IEEE Wireless Communications, vol. 22, pp. 92-99, 2015

[42] N. Al-Falahy and O. Y. Alani, "Technologies for 5G networks: challenges and opportunities," IT Professional, vol. 19, pp. 12-20, 2017.

[43] N. Al-Falahy and O. Y. Alani, "The impact of base station antennas configuration on the performance of millimetre wave 5G networks," in Ubiquitous and Future Networks (ICUFN), 2017 Ninth International Conference on, 2017, pp. 636-641.

[44] F. Khan, Z. Pi, and S. Rajagopal, "Millimeter-wave mobile broadband with large scale spatial processing for $5 \mathrm{G}$ mobile communication," in Communication, Control, and Computing (Allerton), 2012 50th Annual Allerton Conference on, 2012, pp. $1517-1523$.

[45] A. Sayeed, J. Brady, P. Cheng, and U. Tayyab, "Indoor Channel Measurements Using a $28 \mathrm{GHz}$ multi-Beam MIMO Prototype," in Vehicular Technology Conference (VTC-Fall), 2016 IEEE 84th, 2016, pp. 1-5.

[46] R. A. Shafik, M. S. Rahman, and A. R. Islam, "On the extended relationships among EVM, BER and SNR as performance metrics," in Electrical and Computer Engineering, 2006. ICECE'06. International Conference on, 2006, pp. 408-411.

[47] D. Tse and P. Viswanath, Fundamentals of wireless communication: Cambridge university press, 2005.

[48] L. Reznik, Fuzzy controllers handbook: how to design them, how they work: Newnes, 1997.

[49] M. K. a. E. Akin, "Block Based Fuzzy Controllers," IJRRAS, vol. 3 , 2010.

[50] M. Torabi and C. Nerguizian, "Impact of antenna correlation on the BER performance of a cognitive radio network with Alamouti STBC," IEEE Wireless Communications Letters, vol. 5, pp. 264$267,2016$.

[51] C. Schurgers, O. Aberthorne, and M. Srivastava, "Modulation scaling for energy aware communication systems," in Proceeding of the 2001 international symposium on Low power electronics and design, 2001, pp. 96-99.

[52] J. G. Proakis, "Digital communications. 1995," McGraw-Hill, New York. 